\title{
An Extended Isgur-Paton Model: Agreement With the Lattice?
}

\author{
R. W. Johnson* and M. Teper ${ }^{\mathrm{a}}$ \\ ${ }^{a}$ Department of Physics, University of Oxford, Theoretical Physics, \\ 1 Keble Road, Oxford OX1 3NP, United Kingdom
}

The spectrum for the pure gauge sector is calculated for an extended Isgur-Paton model in $2+1$ and $3+1$ dimensions and compared to recent lattice calculations of the glueball spectrum. The IP model is extended by inclusion of a rigidity (curvature) term and, in $\mathrm{D}=2+1$, mixing through a higer topological contribution. For a choice of parameterizations, near quantitative agreement is found for $\mathrm{SU}(3)$ in $\mathrm{D}=2+1$, but in $\mathrm{D}=3+1$ the extensions fail to remedy the qualitative disagreement.

\section{Introduction}

The traditional Isgur-Paton model [1] was inspired from the strong-coupling limit of lattice QCD. It describes a pure glue state as a closed loop of chromoelectric flux of vanishing diameter. The model is nonrelativistic and invokes an adiabatic limit such that motion is split between "fast" transverse oscillcations of the string and "slow" radial excitations. For $S U(N \geq 3)$ the string carries an orientation vector. The predictions of the model depend greatly on the number of spatial dimensions, and so we will discuss the $\mathrm{D}=2+1$ case primarily. Adiabatically separating $\rho_{0}$ allows quantization of the vibrational modes into phonons characterized by quantum occupation number $n_{m}^{ \pm}$, where \pm indicates the "helicity" of the mode. We also should expect a contribution from the rest energy of the string, proportional to the string tension $\sigma$. For a loop of radius $\rho_{0}$, we have the Hamiltonian

$H\left(\rho_{0}\right)=2 \pi \sigma \rho_{0}+\frac{\sum_{i, m} \pi_{i, m}^{2}}{2 \pi \sigma \rho_{0}}+\frac{\pi \sigma}{2 \rho_{0}} \sum_{i, m} m^{2} q_{i, m}^{2}$

where $q_{i, m}$ represents the normal coordinates for $\alpha_{m}$ and $\beta_{m}$ and $\pi_{i, m}$ is the conjugate momentum. The zero-mode energy diverges but may be regularized by imposing a short-scale cutoff $a$ on the string. Such a discretization leads to a zero-mode contribution as $\frac{8 \rho_{0}}{a^{2}}-\frac{13}{12 \rho_{0}}+\cdots$. The

\footnotetext{
*Talk presented at Lattice 97 by R. W. Johnson. Sponsored by the Rhodes Trust.
}

term linear in $\rho_{0}$ can be identified with a "renormalization" of the string tension $\sigma$. The term linear in $1 / \rho_{0}$ has been identified as a Lüscher correction term, but inclusion of a rigidity term before quantization provides a $1 / \rho_{0}$ dependence, and thus this term can be more easily identified with a renormalization of the effective elasticity in the extended model. The phonon modes can be shown to contribute to the energy as $m\left(n_{m}^{+}+n_{m}^{-}\right) / \rho_{0}$, giving the mode energy factor $M=\sum_{m} m\left(n_{m}^{+}+n_{m}^{-}\right)$. For $\mathrm{D}=2+1$, all angular momentum arises from the phonons, thus we have $J=\Lambda=\sum_{m} m\left(n_{m}^{+}-n_{m}^{-}\right)$.

Since we expect the picture of the flux tube to break down near the origin, as well as to soften the singularity in the potential at $\rho_{0}=0$, the potential in our effective Hamiltonian will be multiplied by a heuristic suppression factor, $F(\rho)$. Originally in [1], $F(\rho)=\left(1-e^{-f \sqrt{\sigma} \rho}\right)$, where $f$ is a free parameter near unity, but we find that a rational function, $F(\rho)=(1-f /(\rho+f))$, gives a better fit to the lattice data.

\section{Inclusion of Elasticity}

Both intuition and calculation conclude that a flux tube should have nonvanishing thickness [2] in the transverse dimension. One manifestation of finite thickness we may expect to find even in our nonrelativistic model is an effective elasticity of the flux tube. The free energy per unit length of a rigid bent rod is $\frac{1}{2} \mathcal{Y} \frac{1}{r^{2}} I$, where $\mathcal{Y}$ is the Young's modulus and $I$ is the moment of in- 
tertia perpendicular to the axis of bending. We expect $\mathcal{Y}$ and $I$ to be constant along the flux tube, thus integrating along the length of the flux tube $\oint \frac{1}{2} \mathcal{Y} I \frac{1}{\rho_{0}^{2}} d l \propto \frac{1}{\rho_{0}}$. In string language this is the curvature term. The constant of proportionality is our effective elasticity, denoted $\gamma$, and will be a free parameter in our action. We can see now that the effect of discretization on a scale $a$ is to renormalize the elasticity $\gamma \rightarrow \gamma-\frac{13}{12}$. There has been some discussion [3] as to the sign such an elasticity should take. We leave the elasticity as a free parameter letting the best fit parameter indentify the correct sign.

\section{Mixing}

Under charge conjugation, $C$, the loop orientation flips. To split the $C=+$ and $C=-$ sectors, we must introduce mixing between "left" and "right" orientated loops. We explore two scenarios: direct mixing and mixing through a higher topological state. Writing the independent orientations of the glue loop as $\psi_{L}$ and $\psi_{R}$, the direct mixing matrix Hamiltonian is given in equation (2). For $\Psi=\left(\begin{array}{ll}\psi_{L} & \psi_{R}\end{array}\right)^{T}$,

$\Psi^{\dagger} H_{1} \Psi=\Psi^{\dagger}\left[\begin{array}{cc}H_{L, R} & -\alpha \\ -\alpha & H_{L, R}\end{array}\right] \Psi$,

where $\alpha$ is the mixing parameter and $H_{L, R}$ is the Hamiltonian for a single glue loop. Since we would expect mixing to occur more easily for smaller loops, $\alpha$ may be multiplied by the complement of the suppression function of the potential, so that the mixing goes as $\alpha(1-F(\rho))$. Eigenstates for $C$ are built as $\psi_{L}+\psi_{R}$ and $\psi_{L}-\psi_{R}$.

We may instead include a state, denoted $\psi_{Y}$, consisting of a glue loop with an extra diameter as an intermediate state through which one orientation may fluctuate to the other. Our matrix Hamiltonian then reads, for $\Psi=$ $\left(\begin{array}{ccc}\psi_{L}+\psi_{R} & \psi_{L}-\psi_{R} & \psi_{Y}\end{array}\right)^{T}$,

$\Psi^{\dagger} H_{2} \Psi=\Psi^{\dagger}\left[\begin{array}{ccc}H_{L, R} & 0 & \alpha \\ 0 & H_{L, R} & \eta_{C} \alpha \\ \alpha & \eta_{C} \alpha & H_{Y}\end{array}\right] \Psi$,

where the factor $\eta_{C}= \pm 1$ arises from coupling the $\psi_{Y}$ state to the appropriate admixture of $\psi_{L}$ and
$\psi_{R}$ to match the $\mathrm{C}$ eigenvalue. Since the $\psi_{Y}$ state is not rotationally invariant, we must write the linear superposition as $\psi_{Y}=\int d \theta e^{i J \theta}\left|\psi_{Y \theta=0}\right\rangle$. A quick calculation shows that the $\psi_{Y}$ state has $\mathrm{C}$ eigenvalue $C_{Y}=(-1)^{J}$. The combinations $\psi_{L} \pm$ $\psi_{R}$ have opposite $\mathrm{C}$ eigenvalues, thus by including the factor $\eta_{C}=(-1)^{J}$ the $\psi_{Y}$ state decouples from the admixture with the wrong $\mathrm{C}$ eigenvalue. As above, $\alpha$ may multiply a function of $\rho$, but the energetic cost of creating the diameter for large $\rho_{0}$ provides an inherent suppression of mixing for large $\rho_{0}$.

\section{Building the Hamiltonian}

We build our Hamiltonian from (if) invoking an adiabatic separation of the variable $\rho$, as in the case of mesons [1]. When we take $\pi_{\rho_{0}}{ }^{2} \rightarrow-\frac{d^{2}}{d \rho_{0}^{2}}$, we identify our kinetic terms as

$\begin{aligned} H_{\text {kinetic }}^{L, R} & =\frac{-1}{2\left(2 \pi \sigma \rho_{0}\right)} \frac{d^{2}}{d \rho_{0}^{2}} \\ H_{\text {kinetic }}^{Y} & =\frac{-1}{2\left(2 \pi \sigma \rho_{0}+2 v\right)} \frac{d^{2}}{d \rho_{0}^{2}} .\end{aligned}$

The potential $V\left(\rho_{0}\right)$, given by the rest energy of the string and the contributions from the rigidity term and phonon modes, is

$$
\begin{aligned}
V^{L, R} & =2 \pi \sigma \rho_{0}+\frac{M+\gamma}{\rho_{0}} \\
V^{Y} & =(2 \pi+2) \sigma \rho_{0}+2 v+\frac{\gamma}{\rho_{0}} .
\end{aligned}
$$

The term $2 v$ in $V^{Y}$ is a contribution from the vertices each of static energy $v$. Such a contribution may follow from considerations in [4]. We also must include the rotational contribution for $\psi_{Y}$,

$H_{\text {rotational }}^{Y}=\frac{L^{2}}{2 I_{Y}}=\frac{J^{2}}{\left(4 \pi+\frac{4}{3}\right) \sigma \rho_{0}^{3}+2 v \rho_{0}^{2}}$,

but we do not include any phonon contributions.

\section{Results}

The spectrum is calculated by a suitable numerical diagonalization of the Hamiltonian and compared to recent lattice results (see, for example, [5]). We minimize $\chi^{2}$ with respect to the 


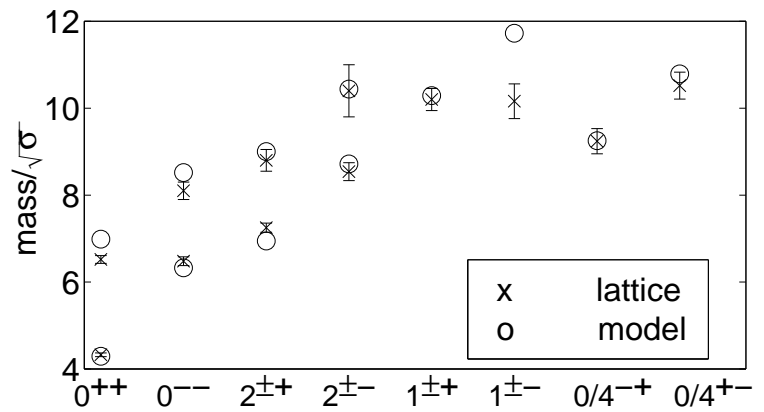

Figure 1. Spectrum for direct mixing mechanism. $f=0.17, \gamma=0.90$, and $\alpha=4.5 . \quad \chi^{2} /$ dof $=$ $44.1 /(12-3)=4.9$.

$J=0,2$ sectors by a simplex search in parameter space and then calculate the full spectrum. Some ambiguity arises when identifying certain $J^{P C}$ states for comparison. Lattice Monte Carlo simulation gives a $0^{-+}$below the $1^{++}$, whereas our model predicts the lightest $0^{-+}$(at $M=8$ ) well above the $1^{++}$. However, our model does predict a $4^{-+}$below the $1^{++}$, suggesting that the Monte Carlo signal may be a $J=4$ in disguise, a possibility for an Euclidean square lattice. Figure 11 summarises our results for the direct mixing mechanism, with $\alpha$ multiplied by $f /(\rho+f)$. Except for the $1^{ \pm-}$, the model and the lattice are in remarkable agreement, although $\chi^{2}$ is somewhat larger than a believable fit should have. The degeneracy of the lattice data for the $J=1$ sector is not available to the model, which maintains a significant splitting for all $J$ such that the $C=+$ states are lighter than the corresponding $C=-$.

For mixing through $\psi_{Y}$, our results are shown in Figure 2, with no multiplicative suppression of $\alpha$. Generally we find a poorer fit despite the additional parameter. Here we notice an interesting feature of this mechanism, namely that for even $J$ the $C=+$ states are lighter, but for odd $J$ the $C=-$ states are lighter. For the $J=1 \mathrm{sec}-$ tor the lattice data must be averaged across parity to make our comparison, and the $1^{ \pm-}$might move relative to the $1^{ \pm+}$as better data become available. Which direction the $1^{ \pm-}$moves (if at all) would provide a reasonable criteria for distinguishing between mixing mechanisms.

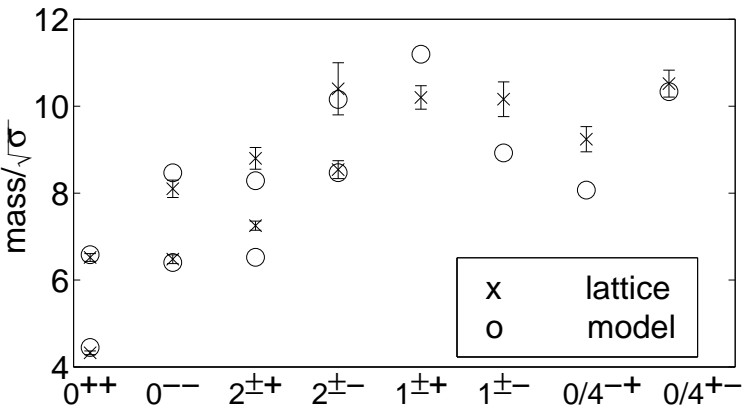

Figure 2. Spectrum for $\mathrm{Y}$ state mixing mechanism. $f=0.28, \gamma=2.4, \alpha=3.8$, and $v=9.3$. $\chi^{2} /$ dof $=64.7 /(12-4)=8.1$.

\section{Conclusions}

The Isgur-Paton model, extended by inclusion of an effective elasticity and by various mixing mechanisms, agrees reasonably with the lattice $\mathrm{SU}(3)$ spectrum in $\mathrm{D}=2+1$, suggesting that a flux tube picture for glueballs might be viable there. In $\mathrm{D}=3+1$, a qualitative discrepancy remains, as the model predicts a low-lying $1^{-+}$as an orbital excitation of the ground state $0^{++}$which is at odds with the relatively heavy $1^{-+}$on the lattice. Nonetheless, the quality of the comparison in $\mathrm{D}=2+1$ suggests futher work be done mapping the pure gauge spectrum for this dimensionality. An interesting point for future comparison would be the mass of the $J=3$ sector, which the model predicts to be signficantly lighter than the $J=1$ sector.

We thank J. Paton for many useful discussions.

\section{REFERENCES}

1. N. Isgur and J. Paton, Phys. Rev. D31 (1985) 2910.

2. C. Y. Wong and G. Gatoff, Phys. Rep. 242 (1994) 489.

3. H. Kleinert and A. M. Chervyakov, hepth/9601030.

4. A. Momen and C. Rosenzweig, Phys. Rev. D56 (1997) 1437.

5. M. Teper, Phys. Lett. B397 (1997) 223. 